\title{
Safe management of bodies of deceased person with suspected or confirmed COVID-19
}

Aabishkar Bhattarai ${ }^{1 *}$, Bijaya Karki $^{2}$

'Medical Officer, Department of Neurosurgery, Manipal Teaching Hospital, Pokhara, Nepal

${ }^{2}$ Medical Officer, Intensive care Unit, Birat Medical College Teaching Hospital, Biratnagar, Nepal

Keywords: Autopsy, COVID-19, Deceased, Safe management

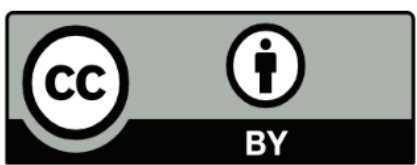

This work is licensed under a Creative Commons Attribution 4.0 Unported License.

\section{Introduction}

Since the detection of the first case of COVID-19 from Wuhan, China, COVID-19 disease is spreading to most of the countries of the world with significant mortality (Case fatality rate:1.4\%). ${ }^{1}$ WHO has classified it as a global Pandemic on March 11,2020. ${ }^{2}$ COVID-19 has been a mysterious, new and evolving disease whose source and disease progression is not entirely clear. Til July 16,2020 , the number of deaths attributed to COVID-19 has surpassed $587,327 .{ }^{3}$ Because of limited data and research studies, the transmission of the virus from dead bodies with COVID-19 has not been verified. In Thailand, as per the Journal of Forensic and Legal Medicine, a forensic personnel got infected with the virus after handling the biological sample and corpses with COVID-19. In Nepal, according to Kantipur National Daily published on June 27 , two army personnel involved in handling and management of COVID-19 corpses are infected with COVID-19 virus. ${ }^{5}$

\begin{abstract}
COVID-19 is a respiratory illness caused by COVID-19 the coronavirus that predominantly affects the lungs. Because of limited data and research studies, the transmission of the virus from dead bodies with COVID-19 has not been verified. However, to stay on a safe side, the safety and well-being of everyone who handles the corpses should be prioritized. All the personnel who interact with the dead bodies (Health Care Workers, Ambulance drivers, Mortuary staffs or Burial teams) should apply the standard precautionary measures including Personal Protective Equipment (gown, gloves, and face protection-face mask and face shield if risks of splashes from body fluids are high). Safety precautions should be taken by the forensic personnel involved in the autopsies of the people who died from COVID-19, as the lungs and other organs can still contain a live virus.
\end{abstract}

\section{Discussion}

COVID-19 is a respiratory illness caused by COVID-19 the coronavirus that predominantly affects the lungs. The mode of transmission includes droplets, fomites, and close contacts, with possible spread via feces. ${ }^{6}$ It is not an airborne disease. ${ }^{6}$ There has been general consensus and various public violence in some part of Nepal regarding spread of COVID-19 virus while handling and burying the dead bodies. According to WHO, dead bodies are not infectious, they do not transmit disease except in cases of hemorrhagic fevers (Ebola, Marburg) and cholera. ${ }^{6}$ Only the lungs of the patients with pandemic influenza if handled improperly

\footnotetext{
${ }^{*}$ Corresponding Author:

Dr. Aabishkar Bhattarai

Medical Officer,

Department of Neurosurgery,

Manipal Teaching Hospital, Pokhara, Nepal

Email: aabishkar.bhattarai@gmail.com

ORCID: https://orcid.org/0000-0003-3669-1707
} 
during autopsy can be infectious. ${ }^{6}$ Workers who routinely handle corpses may have the risk of contracting tuberculosis, bloodborne viruses (e.g. hepatitis $B$ and $C$ and HIV) and gastrointestinal infections (e.g. cholera, E. coli, hepatitis A, rotavirus diarrhea, salmonellosis, shigellosis, and typhoid/paratyphoid fevers).? However regarding COVID-19, there are only a few studies that support the transmission of COVID-19 disease from dead corpses to healthy people.

It is unknown whether a dead body with COVID-19 could transmit disease or not. However, to stay on a safe side, the safety and well-being of everyone who handles the corpses should be prioritized. All the personnel who interact with the dead bodies (Health Care Workers, Ambulance drivers, Mortuary staffs or Burial teams) should apply the standard precautionary measures including Personal Protective Equipment (gown, gloves, and face protection-face mask and face shield if risks of splashes from body fluids are high). ${ }^{6}$ The dead body should be efficiently prepared by removing all the intravenous lines, catheter and tubes; containing all the fluid leaking from the body orifices, wrapping the body with cloth (or body bags if chances of excessive body fluid leakage are high), transferring with minimum movement to the mortuary and hasty disposal should be avoided. If a family member or a friend wishes to view the body, they should be allowed maintaining standard precautions at all times including hand hygiene but strict instructions should be given not to touch, hold, or kiss the body. ${ }^{6}$ Those people with age $>60$ years, immunocompromised, children, or with underlying chronic illness like Chronic Lung Diseases, Heart Diseases, Diabetes mellitus should not be allowed to interact with the body directly. ${ }^{6}$ The body could be buried or cremated respecting the dignity of the dead, their cultural and religious traditions. In the case of mass casualties and where identification of victims is no longer possible, burial is preferable to cremation ${ }^{7}$. Those persons involved in placing the body in the grave should also follow standard precautions with PPE, gloves, and strict hand hygiene after burial is complete.

The graveyards should be at least 30 meters away from the sources of drinking water and the grave floors must be at least 1.5 meters above the water table. Surface water from the graveyards should not enter the inhabited areas.?

The funeral ceremonies after the burial which welcomes many well-wishers and relatives should be postponed till the pandemic is over or if not possible, the number of participants should be kept minimal. Human corona viruses can remain infectious on inanimate surfaces for up to 9 days. ${ }^{8}$ Surface disinfection with $0.1 \%$ sodium hypochlorite or $62 \%-71 \%$ ethanol significantly reduces coronavirus infectivity on surfaces within 1 min exposure time. ${ }^{8}$ Belongings of the deceased need not to be disposed/burnt but could be disinfected with a solution of $70 \%$ of ethanol or $0.1 \%$ bleach. Clothing should be soaked in hot water with detergents or machine washed with warm water at 60-90 degree Celsius and laundry detergent. ${ }^{9}$

Safety precautions should be taken by the forensic personnel involved in the autopsies of the people who died from COVID-19, as the lungs and other organs can still contain a live virus. $^{10}$ Standard precautions, airway precautions, and contact precautions with appropriate face protection (face shield/goggles and N95 or equivalent mask) should be followed during forensic examinations and autopsy. Appropriate PPEs including scrub suit, long-sleeved fluid-resistant gown, gloves (either two pairs or one pair autopsy gloves), and face shield (preferably) or goggles, and boots should be worn. A particulate respirator (N95 mask or FFP2 or FFP3 or its equivalent) should be used in the case of aerosolgenerating procedures..$^{10}$ Autopsies should be performed in an adequately ventilated rooms with at least $160 \mathrm{~L} / \mathrm{s} /$ patient airflow or rooms with negative pressures with at least 12 air changes per hour and mechanical ventilators with the controlled direction of airflow. ${ }^{11}$ Only a minimum number of staff should be involved in the autopsy to minimize the risk of transmission at its most.

\section{Conclusion}

All the health care institutions, health care workers and people involved in managing COVID-19 cases and corpses should follow WHO's WASH practices- provision of safe water, appropriate sanitation, hand hygiene and health care waste management across all parts of the health facility and COVID-19 living and/or dead management areas. ${ }^{9}$

\section{References}

1. 1. Coronavirus Death Rate (COVID-19) - Worldometer. Accessed June 24, 2020. https://www.worldometers.info/ coronavirus/coronavirus-death-rate/

2. WHO announces COVID-19 outbreak a pandemic. Published online March 12, 2020. Accessed June 28, 2020. https://www.euro.who.int/en/health-topics/healthemergencies/coronavirus-covid-19/news/news/2020/3/ who-announces-covid-19-outbreak-a-pandemic

3. Worldometer. Coronavirus Cases. Worldometer. doi:10.1101 /2020.01.23.20018549V2

4. Sriwijitalai W, Wiwanitkit V. COVID-19 in forensic medicine unit personnel: Observation from Thailand. J Forensic Leg Med. 2020;72. doi:10.1016/j.jflm.2020.101964

5. sf]/f]gfaf6 d[To' ePsfsf] zj Joj:yfkgdf vl6Psf b'O\{ hgf ; $\} l g s d f$ ;+qmdOf- ;dfrf/-sflGtk'/ ;dfrf/ Accessed June 27, 2020. https://ekantipur.com/news/2020/06/27/15932351876067 1652.html

6. WHO. Infection Prevention and Control for the safe management of a dead body in the context of COVID-19. J Hosp Infect. 2020;104(3):246-251. doi:10.1016/j. jhin.2020.01.022

7. World Health Organization. Risks posed by dead bodies after disasters. Wer. 2005;3(80):21-28.

8. Kampf G, Todt D, Pfaender S, Steinmann E. Persistence of coronaviruses on inanimate surfaces and their inactivation with biocidal agents. J Hosp Infect. 2020;104(3):246-251. doi:10.1016/j.jhin.2020.01.022

9. WHO. Water, sanitation, hygiene and waste management for the COVID-19 virus. World Heal Organ. 2020;(March):1-9.

10. COVID-19Guidance Postmortem Specimens/CDC. Accessed June 28, 2020. https://www.cdc.gov/coronavirus/2019ncov/hcp/guidance-postmortem-specimens.html

11. Atkinson J, Chartier Y, Lúcia Pessoa-Silva C, Jensen P, Li Y, Seto W-H. Natural Ventilation for Infection Control in HealthCare Settings WHO Library Cataloguing-in-Publication Data: Natural ventilation for infection control in health-care settings. Geneva WHO;. 2009;1. 